8. Lau SK, Chu PG, Weiss LM. CD163: a specific marker of macrophages in paraffin-embedded tissue samples. Am J Clin Pathol 2004; 122: 794801.

DOI: https://doi.org/10.1016/j.pathol.2020.11.013

\section{FNBP1-BRAF fusion in a primary melanoma of the lung}

\section{Sir,}

Primary melanomas of the lung (PML) are extremely rare lung cancers with less than 80 cases published in the English literature. $^{1-3}$ Indeed, metastases of cutaneous primary tumours account for most melanomas diagnosed in the lung, while few pulmonary primary cases are expected to derive from the melanocytes of the tracheo-bronchial apparatus. ${ }^{4}$ Limited genetic studies have been performed in this setting. We report a case of PML with a previously undescribed FNBP1-BRAF fusion.

The study was conducted according to the Declaration of Helsinki and was approved by the research ethics committee of the Center Leon Berard, Lyon, France (Ref: L20-72).

A 55-year-old woman, actively smoking (15 pack year) with a previous medical history of multiple sclerosis and arterial hypertension was referred to the pneumology department for the investigation of a para-mediastinal mass of the left lung. This $7 \mathrm{~cm}$ diameter tumour was discovered during medical investigation for recurrent bronchitis (Fig. 1A). A drill biopsy of the pulmonary mass was performed.

Microscopic examination revealed infiltration of the bronchial mucosa by an undifferentiated tumoural proliferation made of small to medium sized cells harbouring high nucleocytoplasmic ratio and numerous mitotic figures frequently associated with necrosis. No infiltration of the surrounding epithelium was evidenced (Fig. 1B,C).

Immunohistochemistry demonstrated focal expression of the S100 protein and SOX10. No expression of MelanA, HMB45, cytokeratins (AE1/AE3), EMA, chromogranin A, synaptophysin, CD45, CD34, CD99 or TTF1 was observed. Proliferation index (Ki-67) was estimated around $20 \%$. Extension work-up including a body scan positron emission tomography (PET) imaging and dermatological examination did not detect any other tumour location. The patient received chemotherapy (Holoxan and doxorubicine) before surgical resection.

Three years later, a superficial centimetric nodule arose on the right arm of the patient. Microscopic examination revealed dermal infiltration by tumour cells with medium size, amphophilic cytoplasm and large nucleus. No junctional component was detected. Immunohistochemistry confirmed expression of S100 (Fig. 1D) and SOX10, while no positivity was observed with the melanocytic markers HMB45, antiMelanA and anti-MITF. Whole exome RNA-sequencing performed in the skin tumour identified a FNBPI ENST00000446176 (e13)-BRAF ENST00000288602 (e9) fusion. Mutations in BRAF, NRAS, C-KIT, TP53 genes or $C R T C$-TRIM11 fusion were not evidenced. Furthermore, the $B R A F$ rearrangement was confirmed using a $B R A F$ fluorescence in situ hybridisation (FISH) break-apart probe in both the lung primary tumour and the skin metastasis that showed an identical unbalanced pattern with gain of the $3^{\prime}$ BRAF red signal in both samples (Fig. 1E).

Additional pulmonary and skin recurrences were detected with the PET scanner 6 years after the initial diagnosis. Biopsy of the skin tumour revealed a similar morphology to that previously described (Fig. 2A,B). Immunohistochemistry revealed expression of PS100, SOX10, MelanA and HMB45 in tumour cells (Fig. 2C-F). Presence of a BRAF fusion in the skin tumour was further confirmed by FISH again with a similar pattern.

The patient was treated with immune checkpoint inhibitors; however, bone and mediastinal lymph node metastases were detected 5 months after the final diagnosis.

Herein, we report a case of PML with a FNBP1-BRAF fusion. PML is an extremely rare tumour which accounts for $0.01 \%$ of primary lung cancers ${ }^{4}$ and metastatic melanoma appears as the main differential diagnosis in this setting. ${ }^{1,2}$ In 1968 Allen and Drash and in 1969 Jensen and Egedorf proposed that recognition of an intraepidermal component within the lung tumour might allow the distinction between primary tumours and metastasis. ${ }^{1,2}$ However, such findings were counteracted by the identification of intraepidermal involvement in pulmonary metastasis of cutaneous melanoma. Therefore, Wilson and Moran later defined PML as: (1) a solitary tumour, (2) malignant melanoma confirmed by immunohistochemistry, (3) no past history of excision for cutaneous, mucosal or ocular melanoma, (4) central pulmonary lesion, and (5) no demonstrable tumour elsewhere at the time of diagnosis. ${ }^{4}$ Accordingly, our case met these criteria at the time of diagnosis and, although a skin tumour was detected 3 years after the identification of the lung tumour, such large delay suggests it is unlikely that this cutaneous tumour was in fact a primary that had given rise to the lung location.

Although PML might initially arise from the bronchial apparatus, it could alternatively be regarded as a metastatic location of a so called malignant melanoma of unknown primary (MUP). ${ }^{6}$ While the origin of MUP is matter of debate, frequent detections of $B R A F, N R A S$ and $N F 1$ mutations, high mutation burden and prominent UV signature $(\mathrm{C}>\mathrm{T}$ transition) strongly argue in favour of a cutaneous origin for most of the MUP cases. ${ }^{7}$ In the PML setting, although no evaluation of the tumour burden and no research for mutational signatures have been conducted, the initial investigations in 10 PML cases (mainly isolated case reports) have not identified mutations in BRAF, C-KIT and NF1 genes. ${ }^{3}$ Therefore, the absence of common oncogenic drivers of cutaneous melanoma in PML suggests an extracutaneous origin for these cases, as similarly observed for mucosal melanomas. ${ }^{8,9}$ By contrast, recent investigations by Yang et al. of 10 additional PML cases revealed high tumour mutation burden, frequent $R A F, N R A S$, $N F 1, K I T$, or $K R A S$ mutations, and dominant UV-signature mutation profile, strongly arguing for a cutaneous primary origin in these 'PML' cases. ${ }^{10}$

In this context, we detected a previously unreported fusion of the $B R A F$ gene in a PML case. Indeed, although much less frequent than $B R A F$ and NRAS mutations, BRAF fusions, with a conserved functional serine-threonin kinase domain, are an alternative mechanism leading to the activation of the MAP kinases pathway. BRAF fusions have been detected in about $0.3 \%$ of solid cancers including pancreatic, lung, thyroid, colorectal, carcinomas and pilocytic glioma. In 


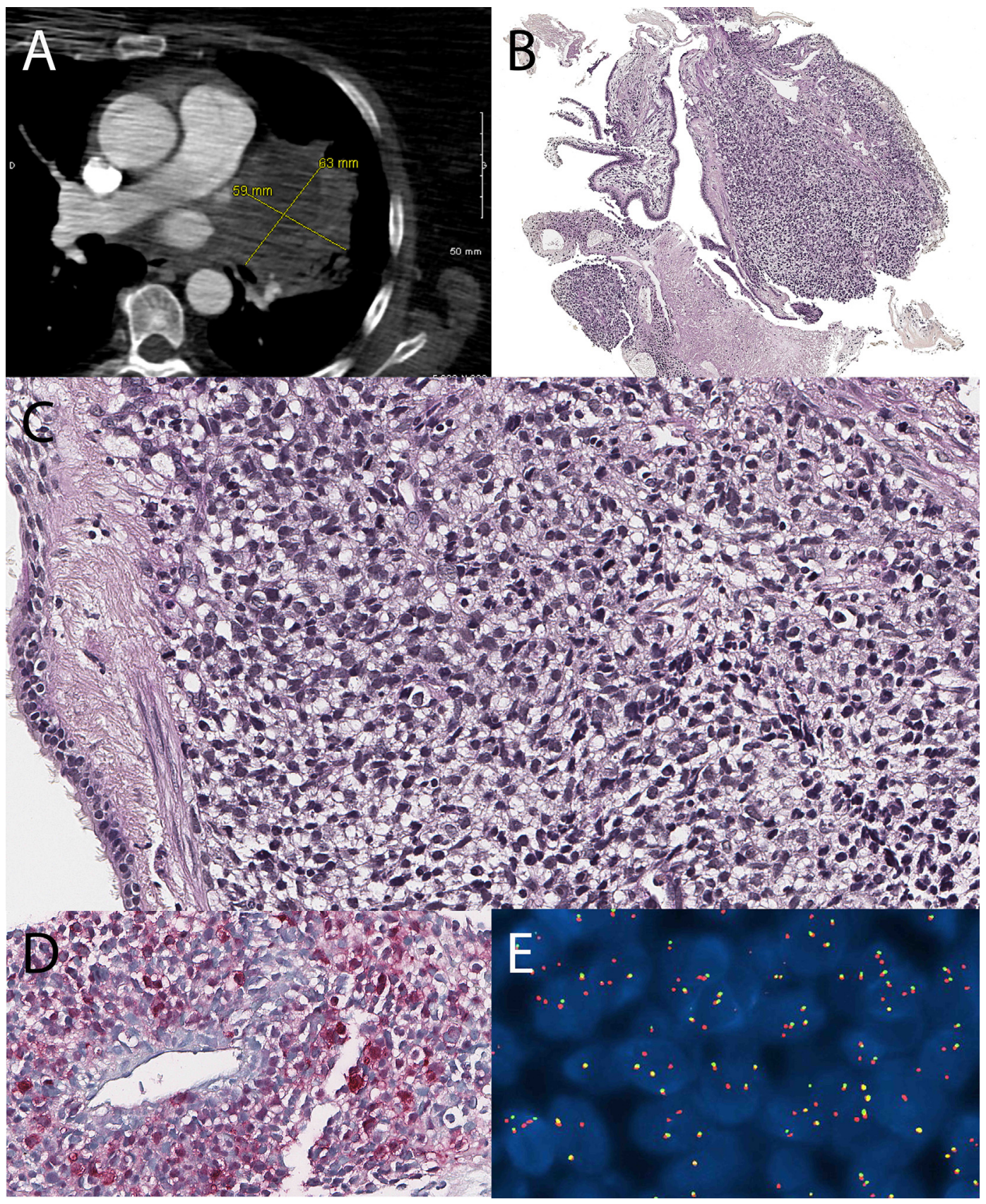

Fig. 1 Imaging, microscopic and molecular features of the pulmonary tumour. (A) Scan imaging revealed a 63 mm para-mediastinal mass of the left lung. (B,C) Microscopic examination of the biopsy confirmed infiltration of the bronchial mucosa by an undifferentiated tumoural proliferation made of small to medium sized cells harbouring high nucleocytoplasmic ratio and numerous mitotic figures frequently associated with necrosis. (D) Immunohistochemistry demonstrated focal expression of the $\mathrm{S} 100$ protein. (E) A BRAF rearrangement was confirmed by FISH using a break apart probe.

melanocytic tumours, such fusions are present in about $5 \%$ of Spitz naevi and are rarely observed in cutaneous melanoma cases. Interestingly, these fusions might have therapeutic implications, since melanoma cases with $B R A F$ fusion were reported to be sensitive to MEK kinases inhibitors but resistant to specific BRAF inhibitors i.e., vemurafemib. ${ }^{1}$

$B R A F$ fusions involve a large number of $5^{\prime}$ partners. Accordingly, a previously unreported $F N B P 1-B R A F$ was detected in our case. FNBP1 (formin binding protein 1) encodes a microtubule binding protein involved in the regulation of cell polarity, motility, and signal transduction, and fusion of $F N B P 1$ with partners other than $B R A F$ were detected in myeloid acute leukaemia. ${ }^{12}$ Therefore, it is likely that FNBP1-BRAF fusion via the activation of the MAP kinases pathways led to melanoma development in our case. 


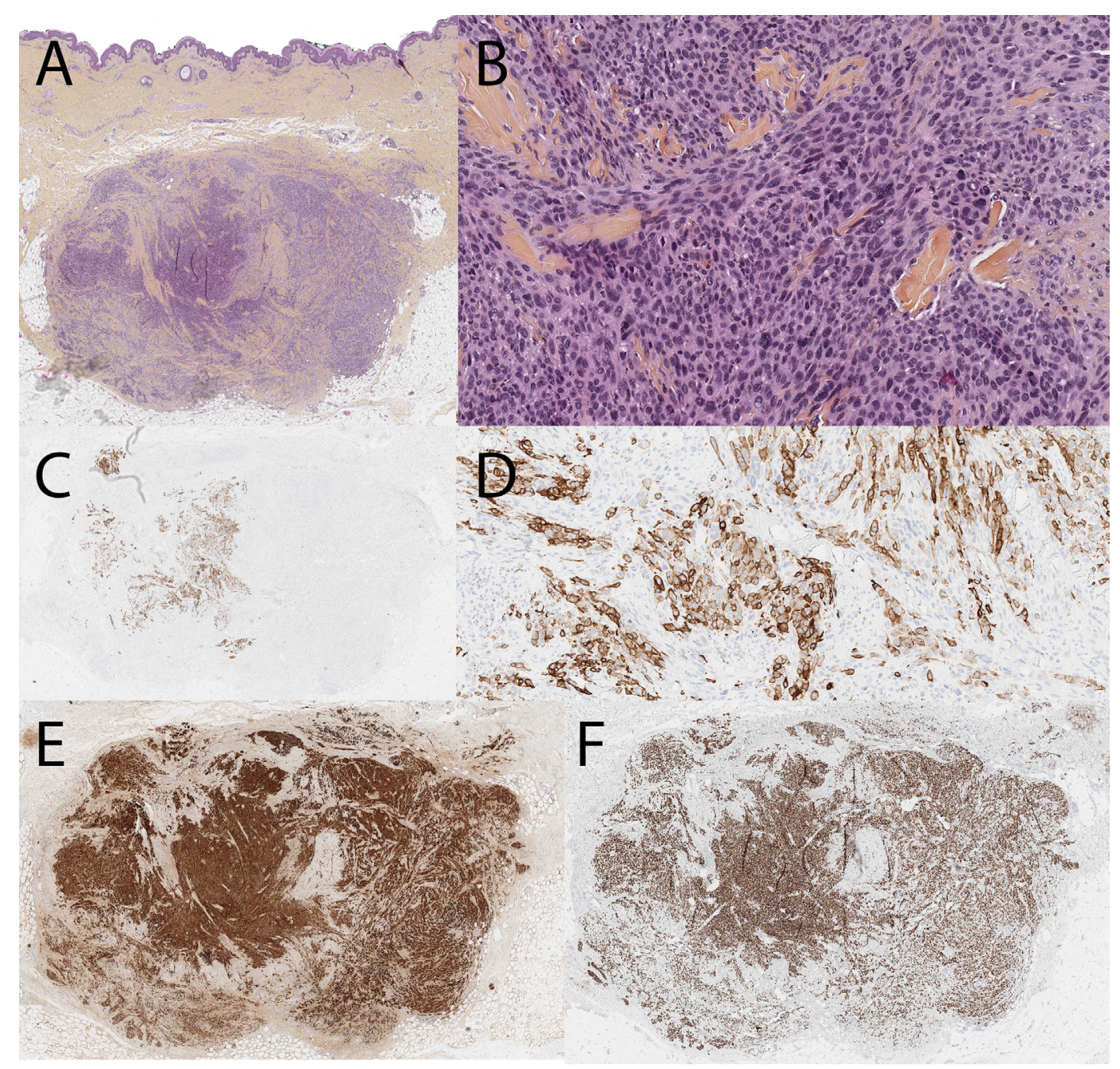

Fig. 2 Morphological and immunohistochemical features of the dermal metastasis. (A,B) Histological examination of the tumour reveals dermal infiltration by tumour cells with medium sized, amphophilic cytoplasm and large nucleus. No junctional component was detected. (C-F) Immunohistochemical investigation of the case demonstrated positivity of tumour cells for HMB45 (C,D), as well as expression of S100P (E) and SOX10 (F).

To conclude, we report a case of PML devoid of the genetic alterations commonly observed in cutaneous melanomas but harbouring an FNBPI-BRAF fusion. Such observation might suggest that specific oncogenic drivers, distinct from those observed in cutaneous melanoma, drive PML development.

Acknowledgements: The authors thank Ms C. Taillandier for FISH illustrations as well as Elise Malandin and Cyril Pi for the immunohistochemistry performed. They also thank Vincent Le Pennec, Jean-Pierre Pelage and François Le Loarer for their help and contribution.

Conflicts of interest and sources of funding: The authors state that there are no conflicts of interest to disclose.

\section{Thibault Kervarrec $^{1,2}$, Bastien Jean-Jacques ${ }^{3}$, Daniel Pissaloux ${ }^{2,4}$, Franck Tirode ${ }^{2,4}$, Arnaud de la Fouchardière ${ }^{2,4}$}

${ }^{1}$ Department of Pathology, Centre Hospitalier Universitaire de Tours, Tours, France; ${ }^{2}$ Department of Biopathology,
Center Leon Berard, Lyon, France; ${ }^{3}$ Department of Pathology, Centre Hospitalier Universitaire de Caen, Caen, France; ${ }^{4}$ Université de Lyon, Université Claude Bernard Lyon 1, INSERM 1052, CNRS 5286, Centre Léon Bérard, Cancer Research Center of Lyon, Equipe Labellisée Ligue contre le Cancer, Lyon, France

Contact Dr Arnaud de la Fouchardière.

E-mail: arnaud.delafouchardiere@1yon.unicancer.fr

1. Allen MS, Drash EC. Primary melanoma of the lung. Cancer 1968; 21 154-9.

2. Jensen OA, Egedorf J. Primary malignant melanoma of the lung. Scand J Respir Dis 1967; 48: 127-35.

3. Hibiya T, Tanaka M, Matsumura M, et al. An NRAS mutation in primary malignant melanoma of the lung: a case report. Diagn Pathol 2020; 15: 11 .

4. Wilson RW, Moran CA. Primary melanoma of the lung: a clinicopathologic and immunohistochemical study of eight cases. Am J Surg Pathol 1997; 21: 1196-202.

5. Littman CD. Metastatic melanoma mimicking primary bronchial melanoma. Histopathology 1991; 18: 561-3.

6. Scott JF, Conic RZ, Thompson CL, Gerstenblith MR, Bordeaux JS Stage IV melanoma of unknown primary: a population-based study in the United States from 1973 to 2014. J Am Acad Dermatol 2018; 79: $258-65$. 
7. Dutton-Regester K, Kakavand H, Aoude LG, et al. Melanomas of un known primary have a mutation profile consistent with cutaneous sunexposed melanoma. Pigment Cell Melanoma Res 2013; 26: 852-60.

8. Newell F, Kong Y, Wilmott JS, et al. Whole-genome landscape of mucosal melanoma reveals diverse drivers and therapeutic targets. Nat Commun 2019; 10: 3163.

9. Hintzsche JD, Gorden NT, Amato CM, et al. Whole-exome sequencing identifies recurrent SF3B1 R625 mutation and comutation of NF1 and KIT in mucosal melanoma. Melanoma Res 2017; 27: 189-99.

10. Yang C, Sanchez-Vega F, Chang JC, et al. Lung-only melanoma: UV mutational signature supports origin from occult cutaneous primaries and argues against the concept of primary pulmonary melanoma. Mod Pathol 2020; 33: 2244-55.

11. Hutchinson KE, Lipson D, Stephens PJ, et al. BRAF fusions define a distinct molecular subset of melanomas with potential sensitivity to MEK inhibition. Clin Cancer Res 2013; 19: 6696-702.

12. Krumbholz M, Jung R, Bradtke J, Reinhardt D, Stachel D, Metzler M Response monitoring of infant acute myeloid leukemia treatment by quantification of the tumor specific MLL-FNBP1 fusion gene. Leuk Lymphoma 2015; 56: 793-6.

DOI: https://doi.org/10.1016/j.pathol.2020.12.009

\section{Sialadenoma papilliferum of the bronchus: focus on histological and genetic distinction from mixed squamous cell and glandular papilloma}

Sir,

Sialadenoma papilliferum (SP) is a rare salivary gland tumour consisting of both an exophytic projection of squamous epithelium and an inverted papillary proliferation of glandular epithelium. ${ }^{1}$ SP usually arises in the intraoral minor salivary glands and rarely occurs in the bronchus. To our knowledge, there are four English case reports on SP of the bronchus or trachea. ${ }^{2-5}$ However, some tumours designated as bronchial SPs in previous publications appeared to represent mixed squamous cell and glandular papilloma (MP); judging from published illustrations, endobronchial prominent papillary structure with a broad fibrovascular core, that is characteristic for MP, was observed in some tumours. Conversely, in a recent publication, a bronchial tumour showing SP-like morphology was described as MP. ${ }^{6}$ No publications in the relevant literature have focused on the histological distinction between SP and MP. We herein report a case of SP of the bronchus and discuss the histological and genetic distinction between SP and MP.

The patient was a 63-year-old man with a history of coughing. Bronchoscopy revealed a whitish, lobulated lesion protruding in the lumen of the left main bronchus (Fig. 1A,B). The patient had undergone endoscopic snare tumourectomy following a diagnosis of benign papilloma, likely an MP, by a pathological examination of a biopsy specimen at a previous institute.

Histologically, a papillary squamous surface and a contiguously endophytic ductal proliferation was seen (Fig. 1C). Oncocytic metaplasia was not found. There was no cytologic atypia or necrosis. Immunohistochemically, p63 [4A4, ready-to-use (RTU); Nichirei Biosciences, Tokyo, Japan] was positive in the surface squamous epithelium and basal layer of the ductal component, while SOX10 (BC34, RTU; Nichirei Biosciences, Japan) was positive in the ductal component (Fig. 1D,E). S100 (polyclonal, RTU; Dako, Australia) was focally positive in the ductal component. Overall, the histological and immunohistochemical findings were consistent with SP.

Recurrent oncogenic alterations in $B R A F$, followed by $H R A S$, have been reported in SP of the intraoral minor salivary glands, ${ }^{7,8}$ but a mutation analysis for SP of the bronchus has not been reported. We thus extracted DNA from a formalin fixed, paraffin embedded specimen and performed targeted sequencing using the Ion AmpliSeq custom panel (Thermo Fisher Scientific, USA) for 23 cancer related genes, as described in detail elsewhere. ${ }^{9}$ No putative driver mutations, including $B R A F, H R A S$ and $A K T 1$ mutations, were detected at a mean depth of $\times 3574$. The lack of $B R A F \mathrm{~V} 600 \mathrm{E}$ mutations was also confirmed by immunohistochemistry using BRAF V600E mutation specific antibody (VE1, RTU; Ventana Medical Systems, USA). These genetic results did not appear inconsistent with the histological classification of the present case as SP; indeed, a lack of BRAF and HRAS mutations has been reported in $20 \%$ of SP cases.

No tumour recurrence was found for 9 months after the last follow-up bronchoscopy following tumour resection.

The major differential diagnosis of bronchial SP is MP. Intrabronchial papillary growth and constituent cells of squamous and glandular cells with a layer of basal cells are findings common to SP and MP (Fig. 2A). However, the distribution of squamous and ductal components is arranged with regularity in SP, while the constituent cells are mixed together in MP. Furthermore, ciliated cells are usually intermixed within MP. However, the distinction between SP and MP may be difficult in small biopsy specimens, and an immunohistochemical analysis may need to be conducted to make a correct diagnosis. Consistent with a previous study, in the present case, SOX10 was positive in the ductal component of SP (Fig. 1E). In addition, due to the lack of published studies on SOX10 staining in MP, we examined the SOX10 immunohistochemistry for four MPs in a recently published cohort. ${ }^{10}$ SOX10 was negative in every epithelial component in all four MPs (Fig. 2B), while SOX10 was positive for bronchial glands as an internal control (Fig. 2C,D).

SOX10 is positive for the acini and intercalated ducts of the salivary glands as well as for the tumours derived from these cells. ${ }^{11}$ Therefore, SOX10 positivity in the ductal component of SP may suggest that the component is derived from the intercalated duct of the minor salivary gland and bronchial gland. ${ }^{7}$ In contrast, MP arises not only in the bronchi, but also in the bronchiole without a submucosal bronchial gland. ${ }^{10,12}$ Taken together, these findings along with the SOX10 negativity in MP suggest that MP derives from the surface respiratory epithelium.

S100 is another marker for the ductal component of SP. However, S100 appeared to exhibit lower sensitivity for the ductal component of SP than SOX10. Consistent with previous studies, ${ }^{3,7}$ the ductal component in the present case of SP showed diffuse and robust positivity for SOX10 but focal positivity for S100. Additionally, we examined the S100 immunohistochemistry for four MPs. S100 was negative in every epithelial component of MPs, while variable numbers of S100-positive dendritic cells were intermixed within the papillomas. These dendritic cells were negative for SOX10. Therefore, these findings suggest that SOX10 could be a 